\title{
Mini-commentary on BJOG-20-0399.R1
}

\author{
T Justin Clark ${ }^{1}$ \\ ${ }^{1}$ Birmingham Womens Hospital
}

June 25, 2020

We are all likely to need a blood test, a biopsy or wind up on the wrong end of an endoscope. We know that the experience is not going to be pleasant. Many of us will revert to techniques to divert attention away from painful stimuli, such as counting forwards or backwards in our heads, deep breathing, imagining tranquil places or listening to music. But the paper published in this issue of BJOG by (Neo D et al, BJOG 2020; $\mathrm{xxxx}$ ) shows us how we can distract patients in a more sophisticated way, using virtual reality (VR) technology. The procedure the research groups chose to investigate was outpatient hysteroscopy.

Outpatient hysteroscopy is a key part of contemporary gynaecological practice. The procedure is acceptable to the vast majority of women, but most will experience some pain and, in a small proportion of women, this can be severe (Smith P, et al, BJOG 2019;126:891-899). Thus, outpatient hysteroscopy, being common and potentially painful, is a good health technology to evaluate the impact of VR technology on patient experience

Deo $\mathrm{N}$ et al, conducted a randomised trial of 40 women undergoing outpatient hysteroscopy for a variety of indications and simple therapeutic procedures were allowed such as biopsy, polypectomy and insertion of a Mirena ${ }^{\circledR}$ device. Women were allocated to standard care or "immersive and interactive video content using a portable, standalone VR headset". The latter delivered a "guided relaxation experience" which included viewing an 8-minute, narrated video depicting "a calming rainforest and lake setting with animated wildlife, which could be explored by using the "head-tracker".

The preliminary results are impressive. Reduction in peri-procedural pain was statistically significant but more importantly the effect size of a $2 \mathrm{~cm}(20 \%)$ difference on a $10 \mathrm{~cm}$ visual analogue scale must be clinically significant. Reduction in anxiety scores were of a similar magnitude. However, the average hysteroscopy procedure duration was less than 4 minutes, which begs the question, is the cost, time and hassle of setting up and using VR technology worth it? Moreover, $16 \%$ of eligible women did not want to use the VR technology because of prior adverse experiences, anxiety or state a preference to see the procedure or use their own distraction media.

No-one is going to change clinical practice on a sample of 40, but many practitioners will be energised to conduct larger scale trials to confirm these provisional results and to analyse more deeply the impact of immersive VR technology on reducing pain and anxiety associated with outpatient hysteroscopy. Future work should look at the type of VR technology, the context where it is deployed for what kind of procedure. The optimal VR programme may vary according to patient characteristics, the type of surgery and its duration. VR technology should be tested in more painful gynaecological interventions such as endometrial ablation, cervical biopsy and transvaginal egg collection. Moreover, the prospective benefit of VR need not be restricted to gynaecological practice but should be evaluated in a whole host of ambulatory procedures involving conscious patients.

No disclosures: A completed disclosure of interest form is available to view online as supporting information. 